\title{
The Effectiveness of Bonded and Vacuum Formed Retainers: A Prospective Randomized Controlled Clinical Trial
}

Niamh O'Rourke ${ }^{a}$, Hussein Albeedh ${ }^{b}$, Pratik Sharma ${ }^{c}$, Ama Johal ${ }^{d}$.

Introduction The objective of this prospective trial was to compare the clinical effectiveness of bonded retainers with vacuum formed retainers, in terms of maintaining the results of orthodontic treatment in the lower arch up to 18 months post debond.

Materials and Methods: This was a hospital-based, prospective randomised controlled clinical trial in which a total of 82 subjects were randomly allocated to one of two groups, receiving either a vacuum formed retainer (Essix Ace Plastic $120 \mathrm{~mm}$ (DENTSPLY)) or a bonded retainer ( 0.0175 coaxial archwire (Orthocare, UK Ltd.) bonded in place with Transbond LR (3M Unitek, UK) for the lower arch. Eligibility criteria included patients nearing debond following treatment with $0.022^{\prime \prime} \times 0.028^{\prime \prime}$ slot size pre-adjusted Edgewise fixed orthodontic appliances whose pretreatment records and study models were available to confirm the presence of pretreatment labial segment crowding or spacing and who had clinically acceptable alignment at the end of treatment. The main outcome was to investigate the clinical effectiveness of the two types of retainers in terms of changes in incisor irregularity at 6 months of retention. The following measurements were recorded at each time point ( 6,12 and 18 months) using a digital caliper: Little's irregularity index, intercanine width, intermolar width, arch length and extraction site opening. A randomization sequence was generated electronically and allocation was concealed with opaque, tamper proof envelopes picked at random by the subject. Blinding was applicable only at T0 due to the permanent nature of one of the interventions.

Results: Both groups were well matched with respect to age, gender, clinical characteristics and treatment plan. There was a statistically significant difference between the groups for changes in Little's Irregularity Index at 6months, with the vacuum formed retainer group showing greater changes than the bonded retainer group ( $P=0.008)$. There was no statistically significant difference between the groups for changes in Little's Irregularity Index at 12 and 18 months.There were also no statistically significant changes at any time period for intercanine width, intermolar width, arch length or extraction site opening.

Conclusions: There is a degree of relapse that is likely to occur following a course of fixed appliance therapy irrespective of retainer choice, and this is minimal in the majority of cases at 6 months after debond. Bonded retainers have a better ability to hold the lower incisor alignment in the first 6 months post treatment when compared with vacuum formed retainers.

Registration: Not applicable.

Protocol: The protocol was not published prior to trial commencement.

Funding: No funding or conflict of interest to be declared 
The goal of orthodontic treatment is to produce an ideal occlusion that is morphologically stable, esthetic and functional ${ }^{1}$. Despite proper diagnosis and carefully rendered treatment mechanics, the results achieved at the end of active treatment are not necessarily stable over the long term.

Post treatment relapse is perhaps the most common risk of orthodontic treatment, and planning for post retention stability should be undertaken as part of the initial treatment plan and discussed with the patient as part of the informed consent process prior to commencing treatment. It presents as a disappointment both for clinician and patient alike.

Stability and relapse, in both treated and untreated malocclusions, have been studied intently over many years ${ }^{2-10}$, and the longterm results have been similar and not hugely optimistic. Sadowsky ${ }^{6}$ followed up patients on average 20 years post retention and found that $9 \%$ had an increase in mandibular crowding when compared to pre treatment and $73 \%$ had dental relationships "outside the norm". Similarly Little ${ }^{10}$ noted that only $10 \%$ of patients had maintained satisfactory mandibular incisor alignment 20 years post retention.

This research serves to demonstrate that the only apparent guarantee of long term stability is long term retention. This is due to the variety of factors which are reported to affect tooth position both in the treated and untreated malocclusion. These are reported to include: skeletal and soft tissue growth ${ }^{11-13}$, dental factors ${ }^{14-16}$, treatment mechanics such as changes in $\operatorname{arch}_{\text {form }}{ }^{17}$, length ${ }^{18}$ and width ${ }^{19}$, treatment plan ${ }^{20-26}$, and final interdigitation ${ }^{27,28}$ and functional occlusion ${ }^{29}$ as well as elements of the pretreatment malocclusion ${ }^{30}$.

Retention is necessary to allow reorganization of the gingival and periodontal tissues affected by orthodontic tooth movement, to prevent unwanted movement as a result from growth changes and to prevent relapse tendency of teeth that have been moved to an inherently unstable position ${ }^{31}$.

Within the United Kingdom the most common types of retention appliances are vacuum formed retainers, Hawley retainers and bonded retainers with the latter being more frequently used amongst private practitioners and the former more commonly prescribed with the National Health Service ${ }^{32}$. A similar study in the United States found an upper Hawley and lower bonded retainer were most popular ${ }^{33}$. Hichens ${ }^{34}$ reported, in a trial carried out in specialist practice within the NHS that a vacuum formed retainer was more preferred by the patients when compared with Hawley retainers and similarly Cerny ${ }^{35}$ identified a patient preference for bonded retainers in private practice. More recently social perceptions of intellectual ability and attractiveness have also been found to be influenced by retainer design and appearance ${ }^{36}$.

Previous prospective research evaluating the clinical effectiveness of removable retention is limited. Of note, in a trial reported on by Rowland ${ }^{37}$ a statistically significant difference was found between the clinical effectiveness of vacuum formed retainers and Hawley retainers, 
with the vacuum formed group being more successful in maintaining post treatment alignment of the anterior teeth after 6 months.

Regarding previous research involving bonded retention this has been mainly retrospective ${ }^{38-}$ ${ }^{41}$ and the majority of prospective studies have investigated failure rates and dental health associated with fixed retainer types as opposed to their clinical effectiveness ${ }^{42-46}$. It was noted though that thin multistranded wires were superior in their ability to maintain lower incisor position $^{44}$.

There is one prospectively designed trial comparing both bonded and vacuum formed retainers up to 24 months post debond ${ }^{47,48}$. In these trials it was reported that a prefabricated positioner used as a retainer showed a statistically significant difference in its inability to maintain incisor position post treatment (measured using Little's irregularity index [LII]) compared with a vacuum formed retainer or a bonded retainer after 6 months ${ }^{47}$ but with no statistically significant difference was found after 2 years ${ }^{48}$.

Retention type and duration of wear is also an ongoing contentious issue within the profession ${ }^{49-52}$. Two Cochrane reviews have been published to date on relapse $\mathrm{e}^{53-54}$ the latest of which reviewed management of relapse and found no study to include in the review. The former review identified limitations to previous research on retention type including short follow up periods, inappropriate or no controls, retrospective in design, insufficient or irrelevant data and thus both highlighted need for randomised control trails in this area to aid in deciding the most effective and safe method for managing the relapse of alignment of the lower front teeth.

The purpose of this study is to quantify and compare the changes in a number of intra-arch variables with vacuum formed retainers and bonded retainers from debond to 6,12 and 18 months and to determine if one type of retainer is superior to the other in terms of maintaining the orthodontic result. These particular retainers have to-date not been directly compared with each other in a randomised controlled trial research study.

\section{Objectives and Null Hypothesis}

The main aim of this randomized controlled trial was to compare the clinical effectiveness of two types of orthodontic retainers in the lower arch in terms of retention of the treated result at 6 months after debond. More specifically, to determine if there are any differences in the clinical effectiveness of vacuum formed retainers (VFR) with bonded retainers (BR) in maintaining the alignment in the lower labial segment (Little's Irregularity Index [LII]) at 6 months after debond. Also, the current study aimed to investigate if there are any differences in the clinical effectiveness of vacuum formed retainers (VFR) with bonded retainers (BR) in maintaining the arch width (intercanine width [ICW]) and intermolar width [IMW]), arch length and extraction site closure. Besides six month time point, 12 and 18 months were also 
considered as previous retrospective studies have shown that the majority of relapse post orthodontic treatment occurs within the first 12 months after debonding of appliances ${ }^{47,48}$.

The null hypothesis which was tested in this study stated that there is no difference in the ability of both types of retainers to retain the treated result in the short or medium term.

\section{Materials and Methods}

\section{Trial Design}

This was a hospital based parallel design randomized controlled clinical trial.

\section{Participants, Eligibility Criteria and Setting}

Ethical approval has been granted by the National Research Ethics Services Committee (REC reference number: 10/H0713/57).

The study population was drawn from patients nearing debond following treatment with $0.022^{\prime \prime} \times 0.028^{\prime \prime}$ slot size pre-adjusted Edgewise fixed orthodontic appliances that fulfilled the inclusion criteria which included:

1. Patients that have completed orthodontic treatment

2. Pre-treatment records and study models were available to confirm the presence of pre-treatment labial segment crowding or spacing

3. Clinically acceptable alignment at the end of treatment

Patients were excluded for the following reasons:

1. Patients who completed treatment early or had repeated breakages during treatment

2. Patients with poor oral hygiene during treatment

3. Patients with prosthodontic needs in the lower arch at end of treatment

4. Patients with a history of periodontal disease

5. Patients with a learning difficulty

Subjects who fulfilled the selection criteria were identified and invited to take part in this study. Informed consent and assent were obtained on the day of debond.

Enrolment started in January 2011 and was completed by February 2012. Eighty five subjects were entered into the trial but during recruitment one declined to take part and 2 further subjects did not meet the inclusion criteria. Therefore, 82 subjects were recruited in total. At the debond appointment after appliance removal (TO) a set of alginate impressions was taken for study models and an additional polysilicone impression of the lower arch was taken in 
order to obtain measurements for the purpose of this study. The polysilicone impression was then cast in hard stone (Type III Stone) in the laboratory on the same day. All subjects were then randomized into their intervention groups.

\section{Interventions}

The vacuum formed retainer was constructed from Essix Ace Plastic (120mm diameter; 0.03inches thick; DENTSPLY, Raintree Essix, Glenroe, USA). This was fabricated by a qualified orthodontic technician under standardised conditions. This was fitted within 7 days with instructions for full-time wear for the first 6 months followed by 6 months of night-time wear and further 6 months of alternate night-time wear which was the recognised department protocol following delivery of retainers.

For the bonded retainer group the teeth were polished with pumice and Cavitron scaling was used if deemed necessary. A 0.0175 inch stainless steel co-axial archwire (Orthocare UK, Saltaire, BD17 7DR, UK) was formed chair side to fit passively against the lower labial segment from canine to canine, using $37 \%$ phosphoric acid etch followed by copious washing, drying and application of an adhesive primer, OrthoSolo bonding agent (Ormco Corporation, California, USA) which was subsequently cured. The wire was then bonded with Transbond LR (3M Unitek, Brackwell, RG12 8HT, UK) composite material.

All subjects were reviewed by a member of the research team (NO'R or HA) and a lower arch dental impression was taken using polysilicone at 6 (T1), 12 (T2) and 18 months (T3) in order to obtain stone models for measurement purposes.

Where patients had lost their retainers, new impressions and retainers were provided. Where there were incidents of appliance breakage or loss, the patients were advised to attend the daily emergency clinic where a new appliance was made, or in the case of a bonded retainer, it was repaired.

\section{Outcome Measurements}

All measurements were performed manually with a digital caliper (150mm DIN 862, ABSOLUTE Digimatic caliper, Mitutoyo Standard Model No: 500-191U, Mitutoyo Ltd, Hampshire, UK) with a resolution of $\pm 0.01 \mathrm{~mm}$. The arch was viewed from above and the caliper held parallel to the occlusal plane and the distance between the contact points of the caliper was recorded.

Irregularity of the mandibular incisors was measured on the study models at pre-treatment, post-treatment (T0) and 6 (T1), 12 (T2) and 18 months (T3) using the same method described by Little ${ }^{55}$ (Figure 1).

Intra arch measurements included the intercanine width (ICW) which was measured as the distance between the two canine cusp tips, and in the case of cuspal wear an estimation of the middle of the surface was made (Figure 2). The intermolar width (IMW) was measured as 
the distance between the mesio buccal cusp tips of both lower first molars and similarly if the cusps tips were worn an estimation of the middle of the surface was made (Figure 2). Arch length was measured at a point midway between the incisal edges of the central incisors, bisecting the line connecting the mesial marginal ridges of the left and right permanent molars (Figure 3).Measurement of the extraction space opening was measured as the sum of contact point displacement in the antero-posterior plane where extractions were carried out.

All model measurements were collected by a single investigator (NO'R). Random errors and intra-operator reliability were assessed by reproducing two sets of measurements on 25 randomly selected study models with a two-week intervening period ${ }^{56}$. Systematic errors were assessed using an intraclass correlation coefficient for repeated measurements.

\section{Sample Size Calculation}

The main outcome measure in this study was change in incisor irregularity within the two groups after 6 months of retention. Sample size estimation, using PASS I I (Hintze, 2011), showed that a total of 72 participants, 36 in each treatment arm, were required to demonstrate a clinically significant difference in the primary outcome of $0.5 \mathrm{~mm}$ between the groups, with $90 \%$ power, SD of 0.79 and alpha of 0.05 , using Mann Whitney $U$ test, and under the null hypothesis $(\mathrm{HO})$ of equal effect. This calculation was based on a previous randomized controlled trial that identified a treatment effect of $0.5 \mathrm{~mm}$ at 6 months follow up ${ }^{37}$. In addition, to allow for a potential $15 \%$ dropout, the sample size was increased to 82 subjects.

\section{Randomization and Blinding}

An electronic randomization programme was used, whereby a subject allocation sequence was generated. The numbers were assigned to either one of the two treatment options to be studied. The random numbers were then placed in blackout envelopes which were all identical, tamper evident and prepared in advance. One envelope was then selected randomly out of many by the patient and opened. 40 subjects were randomized to the vacuum formed retainer group and 42 subjects to the bonded retainer group and all subjects received their allocated intervention.

It was not possible to be blinded to the randomization allocation after TO due to the permanent nature of a bonded retainer. However, measuring the models in a random order and blinding during data analysis aimed to minimise bias.

\section{Statistical Analysis}

Mann Whitney $\mathrm{U}$ tests were performed to allow comparison of the vacuum formed retainer and bonded retainer groups in relation to the different variables at all time periods. A p-value of 0.05 was taken to be statistically significant. 


\section{Results}

\section{Error Study}

Intra-observer reliability was assessed by performing measurements for LII at a 2 week interval on 25 casts $^{56}$ using intra-class correlation coefficients which confirmed excellent agreement between the measures taken with $95 \%$ of the sample demonstrating intraclass correlation coefficient between 0.92 and 0.98

\section{Participant Flow}

The recruitment and follow up of all patients can be seen in the CONSORT flow diagram (Figure 4). Eighty two subjects, mean age 17.73 (3.52 SD), were randomised to either vacuum formed or bonded retainers. At T1 8 subjects failed to attend their appointments at T2 and T3 a further loss to follow up was noted. There was a substantial loss to follow up over all with less subjects returning for their 18 month follow up in the vacuum formed group (60\%) than in the bonded retainer group (74\%).

\section{Baseline Data}

Baseline demographics including age, gender, features of the original malocclusion and treatment plan were similar in both groups. Table 1 shows the mean age and gender distribution and the clinical characteristics of the subjects in their randomised groups.

The treatment details of each group are evident in Table 2. In both groups there were similar amounts of extraction and non extraction treatments. Second premolars were overall the more popular choice for extraction. The amount of crowding was similar in both groups [3.58 (SD 3.3) in BR group and 3.69 (SD 3.94) in the VFR group].

\section{Numbers Analysed for Each Outcome}

For the vacuum formed retainer group, 40, 37, 30 and 24 subject's data was analysed at T0, $\mathrm{T} 1, \mathrm{~T} 2$ and T3 respectively. For the bonded retainer group 42, 37, 34 and 31 subject's data was analysed at T0, T1, T2 and T3 respectively. However to account for the loss to follow up an intention to treat analysis was applied using mean values for the missing data.

\section{Changes in Outcome Measures from T0-T1 (Debond to 6 months post debond) Table 3}

There was a statistically significant difference between the changes observed between the groups at 6 months post debond for Little's Irregularity Index ( $P=0.008$ ).

The median changes in LII, ICW and arch length in the vacuum formed retainer group were higher in this time period than in the bonded retainer group. The difference in the amount of change observed in the latter two outcome measures did not reach statistical significance. A greater change in IMW was observed in the bonded retainer group. There was no significant 
difference between the groups with respect to changes in extraction site space opening over the first 6 months.

\section{Changes in Outcome measures from T1-T2 (6-12 Months post debond) Table 3}

At 12 months there was no statistically significant difference in the amount of change between the groups for any outcome. Extraction site space opening remained minimal in both groups.

\section{Changes in outcome measures from T2-T3 (12-18 months post debond) Table 3}

At 18 months there was no statistically significant difference in the amount of change between the groups for any outcome.

\section{Harms}

There was no harm observed as a result of either intervention. Unlike previous data in the literature $38,40,43$, there was a low failure rate associated with the bonded retainer group over 18 months, with only 3 subjects returning with a single pad which had debonded. This may be due to the fact that the majority (39/42) of bonded retainers were placed by one operator under a standardised protocol.

\section{Discussion}

This randomised clinical trial was designed to investigate the clinical effectiveness of two different retainer types at maintaining the incisor regularity in the mandibular arch, up to 6 months after debond. From an aesthetic standpoint, relapse of the anterior teeth weighs heavily in any assessment of stability of the results, as the patient tends to focus almost exclusively on alignment of the incisors and canines. However, effectiveness could not be based on this alone and therefore additional outcome measures were selected as they indicate the stability of the treatment results and thus the effectiveness of a retention method. These measurements were ICW, IMW, arch length and extraction site space opening and have been routinely utilised in many previous published studies on stability $5,10,23,30,37,43,45,47,50,51$.

One of the advantages of the randomisation process is that it attempts to ensure that confounding variables such as pre treatment malocclusion, irregularity, treatment choice and mechanics are equally divided between the groups so that the groups are equal in all respects except for their intervention type.

In this study the randomisation worked well and the two groups were deemed to be well matched. The age range of the patients in the bonded retention group was slightly higher due to the presence of two 28 year old subjects. There were a higher number of female subjects altogether in the trial and this is a common occurance in orthodontic studies ${ }^{26-39,41,47}$. This may be because females are more self-aware and concerned with dental health than males 
57 and are perhaps more likely to seek treatment for this but also females have a higher desire for orthodontic treatment than males ${ }^{58}$. There was also a higher proportion of Class II Division I malocclusions present and this can be explained by the increased prevalence of this malocclusion in society ${ }^{59}$ as well as in a referred population due to this malocclusion being one of the most common malocclusions that people seek treatment for ${ }^{60}$. The tooth choice for extraction may well be a reflection of the fact that mild and moderate crowding are the most prevalent patterns of crowding both which can be successfully relieved with the extraction of second premolars..

There is a difficulty in directly comparing results of the current study with others, as, although there are similar research questions asked in other studies ${ }^{37,39}$, they are not all similar in their methods $^{37,44,}$ outcome measures ${ }^{42-46,50,51}$ measurement techniques ${ }^{37,39,41}$ and duration of recall $5,23,38,39$. In addition to these issues, others do not display their full results or display results differently $41,45,47,48,54$.

\section{Little's Irregularity Index}

Unlike the findings of previous studies ${ }^{39}$ not all subjects exhibited changes in Little's irregularity index over 18 months after debond. However there was a median increase in Little's irregularity index in both treatment groups over the first six months after debond and the increase reported in the current study was similar to that reported previously regardless of retention regime and type ${ }^{37,42,44-52}$. No patient demonstrated a Little's irregularity index after 18 months of more than $3.5 \mathrm{~mm}$. A score of $<3.5 \mathrm{~mm}$ been deemed as clinically acceptable in other studies $39,47,48,50-52$. Findings for the vacuum formed retainer group reported in the present study are similar to those of Rowland (2007) and Shawesh (2010). The current study figures are more favourable than those reported by Lindauer and Shoff (1998). The findings in the present study for the bonded retainer group are more favourable than those reported by Atack (2007) and Tynelius (2013). The differences may be related to different measuring techniques adopted during the latter studies. Changes of similar magnitudes were documented in longer retrospective studies $38,47,48$.

The findings in this study also suggest that for at least 6 months after debond the bonded retainer is superior to a vacuum formed retainer in maintaining alignment of the lower incisors. Changes in LII over time may be related to remaining growth but may also be due to failure to comply with retention regime, particularly in view of the fact that the vacuum formed retainer is removable. Other reasons may be inadequate fit of the vacuum formed retainer ${ }^{61}$, failure of the bonded retainer to remain in $\operatorname{situ}^{46}$ and thus failure of the retainer to prevent relapse.

\section{Intercanine Width}

There was no statistically significant difference in the amount of change in ICW over the duration of observation between the two groups but this study showed the bonded retainer 
to have less change associated with it during the first 12 months. The size of the change is small and it is not likely to be noted clinically. This is similar to findings of Renkema et al. (2008) who reported that intercanine width was well maintained by the use of bonded retainers. Tynelius et al. (2013) also reported a minimal change in intercanine width in the bonded retainer group. In keeping with the findings of previous studies ${ }^{38,47-48}$, the intercanine width in the vacuum formed retainer group showed a small increase over the study period. In contrast to this, Thickett and Power (2010) reported that intercanine width was well maintained by a vacuum formed retainer over 6 months and 1 year.

\section{Intermolar width}

In the first 12 months post treatment, there was as slightly greater increase in IMW associated with the bonded retainer group and this may be due to the fact that the retainer does not extend to the molar region thus stability in this area is entirely dependent on excellent interdigitation of the buccal segments. The changes were slight in both groups and conclude that the intermolar width was also generally well maintained by both retainer types which is in agreement with findings in other similar studies $39,47,48$.

\section{Arch length}

Arch length changes were equally small. Although the change was greater in the vacuum formed retainer group it was not statistically significant and an explanation for the difference could be related to adherence to the recommended retention regime.

\section{Extraction Site Space}

This has not been well reported in the literature and therefore direct comparison with the findings of the present study is not possible. Extraction site reopening was seen in a small number of patients in both groups (BR group $n=7$, VFR group $n=8$ ) and the increase in space opening was similar for both groups.

In the bonded retainer group, in one case, where space opened by a total of $3.42 \mathrm{~mm}$ this is likely to be clinically significant. One would expect that good buccal interdigitation would enhance the treatment stability (Kahl-Nieke, 1997) however as the periodontal ligament can take up to 232 days to rearrange ${ }^{31}$ it is likely that due to the fact there was no retainer posterior to the canines to help ensure that the periodontal fibres reorganise in the approximated tooth positions, the resultant effect was extraction site reopening. In the VFR group the only true explanation for extraction site space opening is the lack of compliance with retainer wear.

\section{Limitations to this trial}

No account for adherence with retainer wear was assessed in this study and therefore it cannot be confirmed that some of the observed relapse may actually be as a result of non adherence to retention regime rather than a failure in the retainer itself. Previous studies 
which focussed on adherence with different regimes in orthodontics showed various outcomes and highlight the difficulties associated with recording compliance ${ }^{62,63}$. In one such study involving the use of timers to measure wear of an appliance, patients been shown to adhere to their requested regime for slightly over $50 \%$ of the expected duration of wear ${ }^{63}$. Attempts have been made to record compliance with retainer wear but this is usually a subjective assessment of the condition of the retainer at review period or a self assessment by the patient themselves both which can be inaccurate due to the Hawthorne effect or indeed patient or operator judgment bias ${ }^{37,47,48,62}$.

\section{Generalizability}

The generalizability of these results might be limited because this research was undertaken in a single centre hospital service mostly by 1 clinician. The methods and materials are described in detail to aid in similar studies in the future.

\section{Conclusions}

There was a significant difference in incisor irregularity changes at 6 months after debond between the gourps. Thus the null hypothesis can be rejected. Bonded retainers are more effective in their ability to maintain incisor alignment in the lower arch in the first 6 months after debond of fixed appliances when compared with vacuum formed retainers.

All changes that occurred over the first 18 months since debond were indeed minimal in amount. 
Figure 1: Little's Irregularity Index

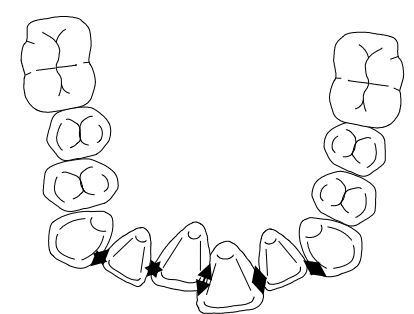

Figure 2: Intercanine and Intermolar Width

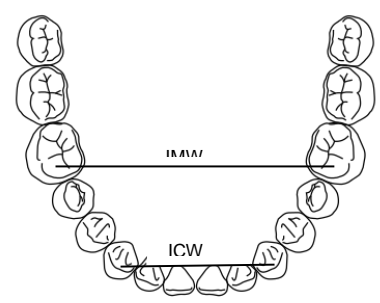

Figure 3: Arch Depth

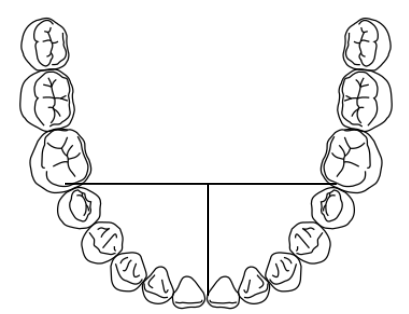


Figure 4 CONSORT Flow Diagram showing subject flow through the trial




Table 1: Demographic and Clinical Characteristics of the Sample at Debond

\begin{tabular}{|c|c|c|c|}
\hline & $\begin{array}{l}\text { Overall sample } \\
\qquad \begin{array}{c}(n=82) \\
\text { Mean (SD) }\end{array}\end{array}$ & $\begin{array}{c}\text { Bonded retainer } \\
\text { sample } \\
\text { ( } n=42) \\
\text { Mean (SD) }\end{array}$ & $\begin{array}{l}\text { Vacuum formed } \\
\text { retainer sample } \\
\text { (n=40) } \\
\text { Mean (SD) }\end{array}$ \\
\hline Age & $17.73(3.52)$ & $18.47(4.41)$ & $16.95(2.02)$ \\
\hline $\begin{array}{l}\text { Gender } \\
\text { Male } \\
\text { Female }\end{array}$ & $\begin{array}{l}23(28.1 \%) \\
59(71.9 \%)\end{array}$ & $\begin{array}{c}9(21.4 \%) \\
33(78.6 \%)\end{array}$ & $\begin{array}{l}14(35 \%) \\
26(65 \%)\end{array}$ \\
\hline $\begin{array}{l}\text { Incisor Classification } \\
\text { Class I } \\
\text { Class II Division } 1 \\
\text { Class II Division } 2 \\
\text { Class III }\end{array}$ & $\begin{array}{l}11(13.5 \%) \\
40(48.8 \%) \\
10(12.2 \%) \\
21(25.5 \%)\end{array}$ & $\begin{array}{c}5(11.9 \%) \\
20(47.6 \%) \\
4(9.5 \%) \\
13(31 \%)\end{array}$ & $\begin{array}{c}6(15 \%) \\
20(50 \%) \\
6(15 \%) \\
8(20 \%)\end{array}$ \\
\hline $\begin{array}{l}\text { Skeletal Pattern } \\
\text { Skeletal I } \\
\text { Skeletal II } \\
\text { Skeletal III }\end{array}$ & $\begin{array}{c}23(28 \%) \\
39(47.6 \%) \\
20(24.4 \%)\end{array}$ & $\begin{array}{l}11(26.2 \%) \\
17(40.5 \%) \\
14(33.3 \%)\end{array}$ & $\begin{array}{c}12(30 \%) \\
22(55 \%) \\
6(15 \%)\end{array}$ \\
\hline $\begin{array}{l}\text { Crowding/Spacing }(\mathrm{mm}) \\
\text { Spacing } \\
\text { No crowding or spacing } \\
\text { Mild } \\
\text { Moderate } \\
\text { Severe }\end{array}$ & $\begin{array}{c}10(12.2 \%) \\
2(2.4 \%) \\
24(29.3 \%) \\
30(36.6 \%) \\
16(19.5 \%)\end{array}$ & $\begin{array}{c}5(11.8 \%) \\
1(2.4 \%) \\
13(31 \%) \\
16(38.1 \%) \\
7(16.7 \%)\end{array}$ & $\begin{array}{c}5(12.5 \%) \\
1(2.5 \%) \\
11(27.5 \%) \\
14(35 \%) \\
9(22.5 \%)\end{array}$ \\
\hline Amount of Crowding (mm) & $3.629(3.62)$ & $3.64(3.25)$ & $3.675(3.8)$ \\
\hline
\end{tabular}


Table 2: Treatment Summary of Sample

\begin{tabular}{|l||c|c|c|}
\hline & Overall sample & $\begin{array}{c}\text { Bonded retainer } \\
\text { sample } \\
(\mathbf{n = 4 2 )} \\
\text { (n=82) } \\
\text { Mean (SD) }\end{array}$ & $\begin{array}{c}\text { Vacuum formed } \\
\text { retainer sample } \\
\text { (n=40) } \\
\text { Mean (SD) }\end{array}$ \\
\hline \hline $\begin{array}{l}\text { Extraction Summary } \\
\text { Extraction }\end{array}$ & $38(46.3 \%)$ & $20(47.6 \%)$ & $18(45 \%)$ \\
Non-Extraction & $44(53.7 \%)$ & $22(52.4 \%)$ & $22(55 \%)$ \\
& & & \\
\hline Extraction Pattern & $14(36.8 \%)$ & $7(35 \%)$ & $7(38.9 \%)$ \\
First Premolars & $16(42.1 \%)$ & $8(40 \%)$ & $8(44.4 \%)$ \\
Second Premolars & $3(7.9 \%)$ & $2(10 \%)$ & $1(5.6 \%)$ \\
Asymmetric Premolars & $5(13.2 \%)$ & $3(15 \%)$ & $2(11.1 \%)$ \\
Other & & & \\
\hline \hline
\end{tabular}

Table 3: Changes in LII, ICW,IMW,Arch length and extraction site space opening at 6,12 and 18 months for Bonded and Vacuum Formed Retainer Groups

\begin{tabular}{|c|c|c|c|}
\hline & $\begin{array}{l}\text { Bonded Retainer } \\
\mathrm{n}=42 \\
\text { (interquartiles) }\end{array}$ & $\begin{array}{l}\text { Vacuum Formed } \\
\text { Retainer } \mathrm{n}=\mathbf{4 0} \\
\text { (interquartiles) }\end{array}$ & $\begin{array}{c}\text { Mann Whitney } \\
\text { P value }\end{array}$ \\
\hline $\begin{array}{l}\text { LII } \\
\text { Change between T0-T1 } \\
\text { Change between T1-T2 } \\
\text { Change between T2-T3 }\end{array}$ & $\begin{array}{l}0.03(0.00-0.07) \\
0.03(0.00-0.06) \\
0.03(0.00-0.10)\end{array}$ & $\begin{array}{l}0.08(0.01-0.31) \\
0.05(0.01-0.20) \\
0.05(0.02-0.18)\end{array}$ & $\begin{array}{l}0.008 \\
0.195 \\
0.300\end{array}$ \\
\hline $\begin{array}{l}\text { ICW } \\
\text { Change between T0-T1 } \\
\text { Change between T1-T2 } \\
\text { Change between T2-T3 }\end{array}$ & $\begin{array}{l}0.11(0.04-0.39) \\
0.17(0.09-0.42) \\
0.17(0.10-0.32)\end{array}$ & $\begin{array}{l}0.23(0.10-0.41) \\
0.20(0.08-0.37) \\
0.26(0.14-0.33)\end{array}$ & $\begin{array}{l}0.214 \\
0.720 \\
0.306\end{array}$ \\
\hline $\begin{array}{l}\text { IMW } \\
\text { Change between T0-T1 } \\
\text { Change between T1-T2 } \\
\text { Change between T2-T3 }\end{array}$ & $\begin{array}{l}0.26(0.10-0.54) \\
0.38(0.75-0.74) \\
0.18(0.70-0.41)\end{array}$ & $\begin{array}{l}0.16(0.06-0.33) \\
0.25(0.88-0.43) \\
0.25(0.13-0.41)\end{array}$ & $\begin{array}{l}0.169 \\
0.565 \\
0.439\end{array}$ \\
\hline $\begin{array}{l}\text { Arch Length } \\
\text { Change between T0-T1 } \\
\text { Change between T1-T2 } \\
\text { Change between T2-T3 }\end{array}$ & $\begin{array}{l}0.19(0.05-0.49) \\
0.20(0.10-0.57) \\
0.18(0.07-0.56)\end{array}$ & $\begin{array}{l}0.23(0.06-0.68) \\
0.19(0.12-0.66) \\
0.19(0.08-0.32)\end{array}$ & $\begin{array}{l}0.512 \\
0.515 \\
0.779\end{array}$ \\
\hline $\begin{array}{l}\text { Extraction site space } \\
\text { Change between T0-T1 } \\
\text { Change between T1-T2 } \\
\text { Change between } \mathrm{T} 2-\mathrm{T} 3\end{array}$ & $\begin{array}{l}0.00(0.00-0.00) \\
0.00(0.00-0.00) \\
0.00(0.00-0.00)\end{array}$ & $\begin{array}{l}0.00(0.00-0.00) \\
0.00(0.00-0.00) \\
0.00(0.00-0.02)\end{array}$ & $\begin{array}{l}0.881 \\
0.799 \\
0.831\end{array}$ \\
\hline
\end{tabular}




\begin{tabular}{|l|l|l|l|}
\hline & & & \\
\hline
\end{tabular}

\section{References}

1. Angle, E.H. Malocclusion of the Teeth 1907; (ed. 7) SS White Dental Manufacturing Company, Philadelphia, PA.

2. Riedel, R.A. A review of the retention problem. Angle Orthod 1960; 30, 179-199.

3. Horowitz, S.L., Hixon, E.H. Physiologic recovery following orthodontic treatment. Am J Orthod 1969; 58, 1-4.

4. Gardner, D.S., Chaconas, S.J. Post treatment and Postretention Changes Following Orthodontic Therapy. Angle Orthod 1976; 46,151-161.

5. Little, R.M., Wallen, T.R., Riedel, R.A. Stability and relapse mandibular anterior alignment- first premolar extraction cases treated by traditional edgewise orthodontics. Am J Orthod 1981: 80, 349-365.

6. Sadowsky C., Sakols, E. I. Long-term assessment of orthodontic relapse. American J Orthod 1982; 82, 456-463.

7. Sinclair, P., Little R. Maturation of untreated normal occlusions. Am J Orthod 1983; 83:114-123

8. Udhe, M.D., Sadowsky C, BeGole E. Longterm Stability of Dental Relationships after Orthodontic Treatment. Angle Orthod 1983; 53, 240-253.

9. Gilmore, C.A., Little, R.M. Mandibular incisor dimensions and crowding. Am J Orthod1984; 86, 493-502.

10. Little, R.M., Riedel, R.A., Artun, J. An evaluation of changes in mandibular anterior alignment from 10 to 20 years postretention. Am J Orthod Dentofacial Orthop 1988; 93, 423-428

11. Behrents, R.G. Growth in the ageing craniofacial skeleton. Craniofacial Growth series 1985. Centre for human growth and development, University of Michigan, Ann Arbor, pp 1-145.

12. Mamandras, A.H. Linear changes of the maxillary and mandibular lips. Am J Orthod 1998; 94: 405-410

13. Nanda, R.S.,Nanda, S.K. Considerations of dentofacial growth in long-term retention and stability: is active retention needed? Am J Orthod Dentofacial Orthop 1992; 101,297-303

14. Richardson, M.E. Late lower arch crowding in relation to primary crowding. Angle Orthod1982; 44, 56-61.

15. Richardson, M. E. Late Lower Arch Crowding in Relation to the Direction of Eruption. Eur J Orthod 1996; 18, 341-347.

16. Peck, S, Peck, H. Crown dimensions and mandibular incisor alignment . Angle Orthod 1972; 42, 148-153. 
17. De La Cruz, A.R,. Sampson P., Little, R.M., Artun, J., Shapiro, P.A. Longterm changes in arch form after orthoodntic treatment and retention. Am J Orthod Dentofacial Orthop $1995 ; 107,518-530$.

18. Bondemark L. Holm, A-K.., Hansen K., Axelsson, S., Mohlin, B., Brattstom, I., Paulin, G.,Pietila,T. Long term stability of orthodontic treatment and patient satisfaction: A systematic review. Angle Orthod 2007; 77, 181-191

19. Burke, S.P., Silveira, A.M., Goldsmith, J., Yancey, J.M., Van S.A., Scarfe, W. A meta analysis of mandibular intercanine width in treatment and postretention. Angle Orthod1997; 68, 53-60

20. McReynolds, D.C., Little, R.M. Mandibular second premolar extraction: Post retention evaluation of stability and relapse. Angle Orthod 1991; 62, 133-144.

21. Haruki, T., Little, R.M. Early versus late treatment of crowded first premolar extraction cases: Postretention evaluation of stability and relapse. Angle Orthod1988; 68, 61-68.

22. Riedel, R.A., Little, R.M., Bui, T.D. Mandibular incisor extraction- postretention evaluation of stability and relapse. Angle Orthod 1992; 62, 103-116.

23. Shields, T. E., Little, R.M., Chapko, M.K. Stability and relapse of mandibular anterior alignment: A Cephalometric appraisal of first premolar extraction cases treated by traditional edgewise orthodontics. A J Orthod 1985; 87, 27-38

24. Mills, J.R.E. The stability of the lower labial segment. A cephalometric survey. Dent Prac 1986; 18: 293-306

25. Paquette, D.E., Beattie, J.R., Johnston, L.E. A longterm comparison of non-extraction and premolar extraction edgewise therapy in "borderline" Class II patients. Am J Orthod Dentofacial Orthop 1992; 102, 1-14

26. Erdinc, A.E., Nanda, R.S., Isiksal, E. Relapse of anterior crowding in patients treated with extraction and non-extraction of premolars. Am J Orthod Dentofacial Orthop 2006; 129, 775-784

27. Andrews, L.F. The six keys to normal occlusion. A J Orthod 1972; 72, 296-309

28. Harris, E.F., Behrents, R.G. The intrinsic stability of Class I molar relationship: A longitudinal study of untreated cases. Am J Orthod Dentofacial Orthop 1988; 94, 6367

29. Weiland, F. The role of occlusal discrepancies in the long-term stability of the mandibular arch. Eur J Orthod 1994; 16, 521-529

30. Little, R.M., Riedel, R.A. Postretention evaluation of stability and relapse- Mandibular arches with generalized spacing. Am J Orthod Dentofacial Orthop Orthopedics 1989; 95, 37-41.

31. Reitan, K. Clinical and histological observations on tooth movement during and after orthodontic treatment. Am J Orthod 1967; 53:721-745.

32. Singh, P., Grammati, S., Kirschen R. Orthodontic retention patterns in the United Kingdom. J of Orthod 2009; 36:115-121 
33. Valiathan, M., Hughes, E. Results of a survey-based study to identify common retention practices in the United States. Am J Orthod Dentofacial Orthop 2010; 137, 170-7

34. Hichens, L., Rowland, H., Williams, A., Hollinghurst ,S., Ewings, P., Clark, S., Ireland,A., Sandy, J. Cost-effectiveness and patient satisfaction: Hawley and vacuum formed retainers. Eur J Orthod 2007; 29, 372-378.

35. Cerny, R., Cockrell, D., Lloyd, D. A survey of patient opinions on fixed vs. removable retainers. J Clin Orthod 2009; 43, 784-7.

36. Meade, M., Millett, D., Cronin, M. Social Perceptions of orthodontic retainer wear. Eur J Orthod 2014; 36, 649-656.

37. Rowland, H., Hichens,L., Williams,A., Hills,D., Killingback,N., Ewings, P., Clark,S., Ireland, A., Sandy, J. The effectiveness of Hawley and vacuum-formed retainers: a single centre randomized controlled trial. Am J Orthod Dentofacial Orthop 2007; 132, 730-7.

38. Renkema, A.M., Renkema, A., Bronkhorst, E., Katsaros, C. Long-term effectiveness of canine-to-canine bonded flexible spiral wire lingual retainers. Am J Orthod Dentofacial Orthop 2011; 139, 614-621

39. Atack, N., Harradine, N., Sandy J., Ireland, A. Which Way Forward? Fixed or Removable Lower Retainers. Angle Orthod 2007; 77, 594-599.

40. Zachrisson, B.U. Long-term experience with direct-bonded retainers: update and clinical advice. J Clin Orthod 2007; 41, 728-737

41. Barlin, S., Smith, R., Reed, R., Sandy, J., Ireland, A.J. A retrospective randomized double-blind comparison study of the effectiveness of Hawley vs vacuum-formed retainers. Angle Orthod 2011; 81 (3), 404-409.

42. Rose, E. F., Sibylle, J., Irmtrud, E. Clinical comparison of a muitistranded wire and a direct-bonded polyethylene ribbon-reinforced resin composite used for lingual retention. Quintessence International 2002; 33:579-583.

43. Årtun, J., Spadafora, A.T., Shapiro, P.A. A 3-year follow-up study of various types of orthodontic canine to canine retainers. Eur J Orthod 1997; 19, 501509.

44. Stormann, I., Ehmer, U. A Prospective Randomized Study of Different Retainer Types. J Orofacial Orthop 2002; 63, 42-50.

45. Tacken, M. E. , Cosyn,J. , De Wilde, P., Aerts,J., Govaerts,E., Bart V. V. Glass fibre reinforced versus multistranded bonded orthodontic retainers: a 2 year prospective multi-centre study. Eur J Orthod 2010; 32 , 117-123.

46. Taner, T., Aksu, M. A prospective clinical evaluation of mandibular lingual retainer survival. Eur J Orthod 2011; 34 , 470-474

47. Tynelius G.E., L Bondemark, I., Lilja-Karlander, E. Evaluation of orthodontic treatment after 1 year of retention-a randomized controlled trial. Eur J Orthod 2011; 32, 542547. 
48. Tynelius G.E., L Bondemark, I., Lilja-Karlander, E. A randomized controlled trial of three orthodontic retention methods in Class 1 four premolar extraction cases- stability after 2 years in retention. Orthod Craniofac Res 2013; 16, 105-115.

49. Lindaur, S.J., Shoff, R.C. Comparison of Essix and Hawley retainers. J Clin Orthod 1998; 2, 95-7.

50. Thickett, E., \& Power, S. A randomized clinical trial of thermoplastic retainer wear. Eur J Orthod 2010; 32, 1-5.

51. Shawesh, M., Bhatti, B., Usmani, T., \& Mandall, N. Hawley retainers full-or part-time? A randomized clinical trial. Eur J Orthod 2009; 32: 165-170

52. Gill, D. S., Naini, F. B., Jones, A., \& Tredwin, C. J. Part-time versus full-time retainer wear following fixed appliance therapy: a randomized prospective controlled trial. World J Orthod 2006; 8, 300-306.

53. Littlewood, S.J., Millett, D.T., Doubleday, B., Bearn, D.R., Worthington, H.V. Retention procedures for stabilising tooth position after treatment with orthodontic braces. Cochrane Database of Systematic Reviews 2006

54. Yu Y, Sun J, Lai W, Wu T, Koshy S, Shi Z. Interventions for managing relapse of the lower front teeth after orthodontic treatment. Cochrane Database of Systematic Reviews 2013

55. Little, R.M. The irregularity index: A quantitative score of mandibular anterior alignment. AM J Orthod 1975; 68, 554-563

56. Houston, W.J.B. The analysis of errors in orthodontic measurements. Am J Orthod 1983; 83, 382-390.

57. Shaw, W. C. The influence of children's dentofacial appearance on their social attractiveness as judged by peers and lay adults. Am J Orthod 1981, 79, 399-415.

58. Harrison, J. E., O'Brien, K. D., \& Worthington, H. V. (2007). Orthodontic treatment for prominent upper front teeth in children. The Cochrane Library.

59. Todd J E, Lader D .Adult dental health 1988 United Kingdom. Her Majesty's Stationery Office, London Kilpenlainen, P.V., Philips, C., Tulloch, J.F. Anterior tooth position and motivation for early treatment. Angle Orthod 1993; 63, 171-174

60. Johal, A., Sharma, N. R., McLaughlin, K., \& Zou, L. F. (2014). The reliability of thermoform retainers: a laboratory-based comparative study. Eur J Orthod 2014; cju075.

61. Pratt, M. C., Kluemper, G. T., \& Lindstrom, A. F. Patient compliance with orthodontic retainers in the postretention phase. Am J Orthod Dentofacial Orthop 2011; 140, 196201.

62. Bartsch, A., Witt, E., Sahm, G., Schneider, S Correlates of objective patient compliance with removable appliance wear. Am J Orthod Dentofacial Orthop 1993;104, 378-386 